\title{
Inserción laboral de egresados de ingeniería agroindustrial bajo la óptica de la teoría del capital humano
}

\section{Labor insertion of graduates of agroindustrial engineering from the perspective of the theory of human capital}

\author{
GONZÁLEZ-PÉREZ, Cándido*† \& GONZÁLEZ-ANAYA, Ana Gabriela \\ Centro Universitario de los Altos de la Universidad de Guadalajara, Av. Rafael Casillas Aceves No. 1200, Centro \\ Universitario de los Altos, 47620 Tepatitlán de Morelos, Jal.
}

ID $1^{\text {er }}$ Autor: Cándido, González-Pérez

ID $1^{\text {er }}$ Coautor: Ana Gabriela, González-Anaya

DOI: $10.35429 / J E T .2019 .10 .3 .17 .26$

Recibido: 24 de Octubre, 2019; Aceptado 29 de Diciembre, 2019

\begin{abstract}
Resumen
Los egresados de la Carrera de Ingeniería Agroindustrial del Centro Universitario de Los Altos provienen de un medio con carencias económicas y una base familiar desfavorable en cuanto al bajo grado escolar que sustentan sus padres. La Teoría del Capital Humano establece entre sus principales preceptos que cada individuo es el responsable directo e irreemplazable de su futuro escolar y laboral, con los resultados que presento en estas líneas se comprueba que los únicos artífices del éxito laboral son los egresados y han utilizado con esmero los conocimientos adquiridos durante el período de su formación académica. Por medio de la aplicación de un método cualitativo, la información que analizo, proviene de entrevistas a la totalidad del grupo de egresados de la generación 2008-2012; corresponde a lo que en los medios académicos se determina como Estudio en Caso y los resultados aquí expuestos no pueden hacerse extensivos a otras generaciones o a egresados de otras carreras. Es deseable que se comparen características con estudios de origen similar, más no transponer conclusiones.
\end{abstract}

Estudiantes, Trabajo, Formación Universitaria, Clase Social, Capital Humano

\begin{abstract}
The Agroindustrial Engineering graduates from Centro Universitario de Los Altos come from an environment with economic deficiencies and unfavorable family basis in terms of the low academic level their parents have. The Human Capital Theory establishes among its main precepts that each individual is directly and irreplaceably responsible for their school and work future. With the results I present in these lines, it is verified that the only architects of their labor success are the graduates themselves, and how they've used the knowledge they acquired during their academic training. Through the application of a qualitative method, the information I present comes from interviews with the entire group of graduates from the 2008-2012 generation. Corresponding to what academics call a Study Case and the results presented here cannot be extended to other generations of graduates from the same or different majors. It is desirable to compare the characteristics with studies of similar origin but not to transpose the conclusions.
\end{abstract}

Graduates, Work, University Education, Social Origin, Human Capital

Citación: GONZÁLEZ-PÉREZ, Cándido \& GONZÁLEZ-ANAYA, Ana Gabriela. Inserción laboral de egresados de ingeniería agroindustrial bajo la óptica de la teoría del capital humano. Revista Teoría Educativa. 2019. 3-10: 17-26.

\footnotetext{
* Correspondencia del Autor (correo electrónico: candido195913@yahoo.com.mx)

$\dagger$ Investigador contribuyendo como primer autor.
} 


\section{Introducción}

El principal objetivo que se pretende cubrir con los resultados de esta investigación, es que los motivos individuales de los egresados de la carrera de Ingeniería Agroindustrial de la generación 2008-2012 fueron decisorios para tener éxito laboral. El universo de estudio está conformado por 17 egresados de los 24 que culminaron la carrera; a algunos no fue posible localizarlos.

El respaldo hipotético que se presenta en estas líneas proviene de la corriente conocida como Teoría del Capital Humano, donde se sostiene que las personas deciden invertirse a sí mismos para convertirse en trabajadores altamente calificados y una vez empleados en condiciones ventajosas de acuerdo al mercado laboral, "recuperar" lo que han dejado de ganar.

Existe otra corriente del pensamiento que niega sean los motivos propios los decisorios en el futuro éxito laboral, y que es el origen y el ambiente familiar el que proporciona (o niega) ser triunfante en el futuro; a esa tendencia se le conoce como la Teoría del Capital Cultural. Sin embargo, lo aquí analizado coincide ampliamente con la Teoría del Capital Humano y es lo que se demostrará renglones abajo.

Con respecto a la exposición, primero se describirán las principales características de la teoría, luego se habla de lo "masculino" de la carrera en la universidad, después la escolaridad de los padres de familia para exponer que no tuvieron gran influencia ni en la elección universitaria y tampoco en su incursión laboral, después se aborda el tema de las posibles deficiencias en la formación universitaria, y finalmente con la explicación de cómo llegaron a conseguir empleo los egresados y las cualidades que más valoran los empleadores de este tipo de profesionistas.

\section{Teoría del Capital Humano}

Cuando terminó la Segunda Guerra Mundial los países iniciaron la reconstrucción y se apoyó la idea de que era necesario aumentar el porcentaje destinado a la educación para dinamizar la restauración, en ese momento dio inicio a lo que se conoce como Economía de la Educación que consiste en suscribir que la mejor inversión que pueda realizar un estado debe destinarse en formar trabajadores calificados.
Se pensaba en esa época que las universidades eran las únicas que podrían ejercer ese privilegio, no se le daba importancia al papel que jugaban las empresas, o en el mejor de los casos, si se aceptaba, no se le comparaba a lo que se hacía en las universidades.

La Teoría del Capital Humano establece que existe una relación directa entre la inversión en la educación superior y la capacitación de los trabajadores, y aún más, cuando se aumenta la inversión en la educación, significa que se sacrifican otros rubros, luego entonces, la oferta de servicios educativos lleva dentro de sí misma un costo de oportunidad (Leyva López y Cárdenas Almagro, 2002).

Existe una máxima en Economía donde se establece que el costo de una cosa es igual al costo de lo que se dejó de comprar, si se destina un millón de pesos en educación, se deja de comprar un millón de pesos en materiales de construcción o de alimentos, etcétera.

El capital adopta tres formas diferentes: dinero, trabajo y mercancías que cubren algún tipo de necesidad; luego entonces, el trabajo se compra como cualquiera otra mercancía (Bowles y Gintis, 1975) pero tiene una característica que la distingue de las otras porque es la única capaz de crear mercancías y lo puede hacer desde un punto de vista de trabajador simple o de trabajador calificado. Para que una persona se transforme de trabajador simple en trabajador calificado, la universidad cuenta con el monopolio para educarlo.

En este último caso es donde centra su análisis la Teoría del Capital Humano y se afirma que existe una relación directa porque a mayor inversión en educación, mayor desarrollo económico (Villalobos Monroy y Pedroza Flores, julio- diciembre, 2009). 
Las personas gastan en sí mismas para satisfacer sus necesidades, se puede comprar un auto, una casa, alimentación, vestimenta, etcétera, o se puede invertir en sí mismo, es decir, puede inscribirse en una universidad, pagar sus colegiaturas, adquirir sus libros y destinar una cantidad muy grande de horas para convertirse en trabajador calificado ${ }^{1}$; este costo de oportunidad que consiste en invertirse a sí mismo en lugar de adquirir satisfactores inmediatos, es el monto económico que según la Teoría del Capital Humano se debe contabilizar para determinar el monto de la Tasa Interna de Retorno que significa el obtener un sueldo mayor al promedio cuando el estudiante se emplea como profesionista porque es especialista y provee de nuevos procesos y técnicas en su espacio de trabajo (Briceño Mosquera, 2011) ${ }^{2}$. Una segunda oleada de los conceptos teóricos tuvo lugar en la década de los ochenta cuando se llegó a plantear que el estado debería invertir cada vez más para aumentar las tasas de escolarización (Aronson, 2007). Una de las principales críticas que se le han hecho al bagaje teórico propuesto por Shultz, es que a la educación no se le puede circunscribir como recipiente de la inversión económica sino que es la formadora especial del desarrollo armónico del individuo y no solo como capacitadora para el trabajo (Garrido Trejo, 2007).

\section{Carrera masculina}

El 55 por ciento de los egresados entrevistados son hombres, la carrera de Ingeniería Agroindustrial continúa siendo masculina en una región donde la Población Económicamente Activa mantiene una disminución sensiblemente menor de los trabajadores del campo con respecto al total del país ${ }^{3}$.

\footnotetext{
${ }^{1}$ En otras palabras, cambian el consumo inmediato por una inversión a largo plazo. De acuerdo con Leyva López y Cárdenas Almagro (2002), el concepto de capital humano como tal, nació en 1962 cuando en una publicación del Journal of Political Economy habló de inversión en seres humanos cuyo autor fue Theodore W. Shultz. Otros autores prefieren diferencias capital humano de "capacidad humana" porque afirman que ésta última categoría es más apropiada pensando en que el trabajo crea "desarrollo" y no solo "crecimiento" (Kumar Sen, 1998).

${ }^{2}$ Becker, continuador de la obra de Shultz, afirma, de acuerdo a Leyva López y Cárdenas Almagro (2002) que "Una inversión en educación equivale a una inversión en una "máquina" adaptada al cuerpo humano que mejora los resultados en el puesto laboral; los rendimientos futuros de esa "máquina" probablemente superarán el gasto de tiempo y dinero que supone su compra."

${ }^{3}$ La tendencia natural es hacia la "terciarización" que consiste en aumentar la cantidad de trabajadores de los servicios y a disminuir los de la industria y del campo; de acuerdo a la información censal del año 2010 del Instituto Nacional de

La región de Los Altos de Jalisco se caracteriza por haberse consolidado como un polo de desarrollo basado en la avicultura y la porcicultura a nivel nacional. En este ámbito, la carrera de Ingeniería Agroindustrial como una oferta educativa del nivel superior se antoja como una de las más adecuadas porque está acorde con los requerimientos productivos. Sin embargo, es de las menos solicitadas por los estudiantes porque subyace la opinión de que si se busca una opción universitaria será para hacer cosas diferentes a las que ya se hacen, se inscribirán en la universidad para convertirse en profesionistas que laboren dentro de una oficina, si toda su familia ha trabajado en el campo, no quieren estudiar para regresar a trabajar al campo. De las 13 opciones de formación profesional que existen en el Centro Universitario de Los Altos, la carrera de Ingeniería Agroindustrial siempre cuenta con los menores puntajes de admisión ${ }^{4}$.

\section{Escolaridad de los padres}

Las actividades económicas que desarrolla el padre o tutor son en su inmensa mayoría relacionadas con la producción del campo: pequeños ganaderos, labradores o comerciantes del ramo agropecuario. La escolaridad es muy baja como puede observarse en la siguiente gráfica, se pudo conocer la existencia de un solo profesionista y la mayoría tuvo acceso a la enseñanza primaria. Como dato adicional, en una pregunta específica durante las entrevistas se les consultó cuántos de sus hermanos habían cursado o estaban cursando el nivel superior de la educación, el promedio de hermanos en total fue de 3 , y de ellos, 0.85 eran o habían sido universitarios.
Estadística y Geografía, el $12.60 \%$ de la Población Económicamente Activa a nivel nacional se localizaban en el sector primario de la economía, mientras que en particular, en la ciudad de Tepatitlán que es la población cabecera de la región de Los Altos de Jalisco y donde se ubica el Centro Universitario que se aplicó la encuesta a los egresados, la cifra es de $18.20 \%$.

${ }^{4}$ Dicho puntaje consiste en obtener la suma del promedio de calificaciones obtenido en el nivel inferior, en este caso la enseñanza preparatoria, y del examen de ingreso. En el caso de los estudiantes que fueron admitidos en el Calendario Escolar 2017 B, la carrera de Abogado tuvo como puntaje mínimo 143.4133, Ingeniería Agroindustrial 139.222, Ingeniería en Computación 143.1022, Ingeniería en Sistemas Pecuarios 123.3333, Cirujano Dentista 158.6400, Administración 143.1744, Contaduría Pública 157.3600, Enfermería 145.8889, Medicina Veterinaria y Zootecnia 151.9044, Negocios Internacionales 147.5278, Nutrición 141.4122, Psicología 145.0000 y Medicina 176.8256

GONZÁLEZ-PÉREZ, Cándido \& GONZÁLEZANAYA, Ana Gabriela. Inserción laboral de egresados de ingeniería agroindustrial bajo la óptica de la teoría del capital humano. Revista Teoría Educativa. 2019 
En realidad son los entrevistados los pioneros familiares en su incursión académica ${ }^{5}$; con respecto a los egresados de otra opción universitaria en el mismo Centro Universitario de Los Altos -Negocios Internacionales-, 1.00 de los hermanos habían sido universitarios, con esto se reafirma que ellos son de los iniciadores en el ámbito familiar (González y Machaen, 2015).

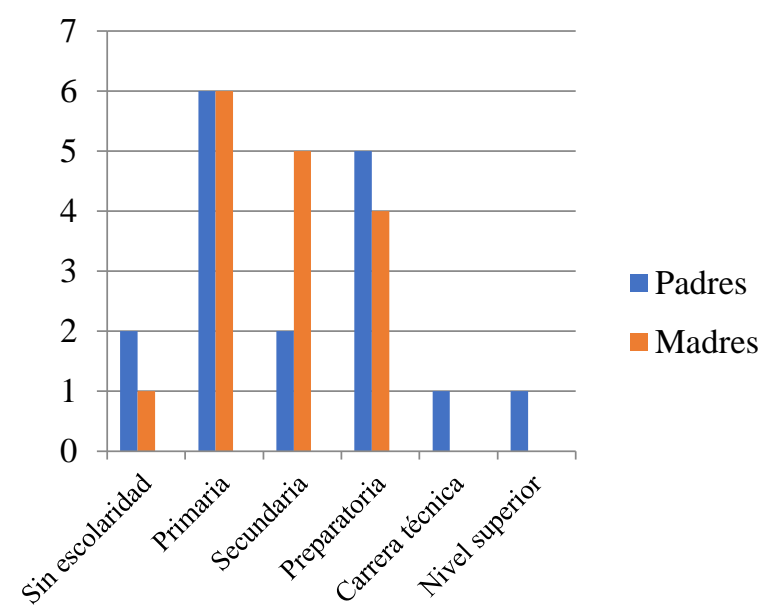

Gráfico 1 Escolaridad de los padres de familia Fuente: información primaria obtenida directamente de las entrevistas

Otra característica del nivel económico que tuvieron siendo estudiantes, es que en las universidades privadas la inmensa mayoría de los alumnos dedica tiempo completo a sus estudios, y el caso de los entrevistados, se vieron obligados a trabajar para poder mantener su inscripción.

A pregunta expresa de cuál fue su medio de sostenimiento financiero, los resultados nos arrojan una proporción muy elevada de que fueron ellos mismos con su trabajo que lo hicieron posible, solamente una proporción muy baja dependió exclusivamente de sus padres. Una proporción muy similar se pudo observar en un trabajo similar que se aplicó a los egresados de la Carrera de Contaduría Pública durante el mismo lapso que se realizó el de Ingeniería Agroindustrial (González y Ortega, 2015).

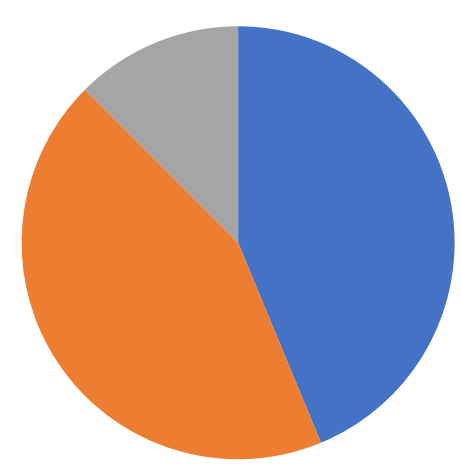

- Con el trabajo de ellos mismos

Con el trabajo de ellos mismos y con apoyo de sus padres

Solamente con el apoyo de sus padres o tutor.

Gráfico 2 Medio de sostenimiento económico durante sus estudios

Fuente: información primaria obtenida directamente de las entrevistas

\section{Empleo de los egresados}

La cualidad más importante de esta investigación por tratarse de la inserción laboral, es la referida a contar o no con un trabajo remunerado, que lo haya obtenido después de haber terminado de la carrera y si la actividad tiene relación estrecha con su formación profesional. Estas características son las que se buscan con más escrupulosidad porque del resultado de su análisis es como se puede comprobar si la Teoría del Capital Humano es lo suficientemente explícita en cuanto a la relación que existe entre el esfuerzo individual y el éxito laboral. Es significativo el mencionar que ninguno de los estudiantes que culminaron su carrera está desempleado, la totalidad cuenta con un contrato laboral.

En el siguiente gráfico se describen las principales categorías para el análisis y destaca en primer orden que la gran mayoría obtuvo su empleo antes de egresar lo que significa que la formación profesional no fue determinante en su contratación, tenemos entonces que los méritos propios son definitorios para la superación. Denomino como categoría especial "influencia individual" al esfuerzo desarrollado por las personas para pertenecer a las filas del mercado laboral, e "influencia profesional" a la que otorga una ventaja a los egresados para obtener un empleo.

\footnotetext{
${ }^{5}$ En términos teóricos se le denomina "principio meritocrático basado en el esfuerzo personal" (Aronson, 2007). 
De los profesionistas que representan a la categoría de la "influencia individual", su inmensa mayoría desempeña actividades relacionadas estrechamente con los saberes ofrecidos en la universidad, y de quienes personifican a la "influencia profesional", son más los que trabajan en áreas que no son concordantes con su especialización.

De lo anterior se pueden extraer tres pruebas concluyentes, primero, si una de las principales finalidades de la existencia de oferta educativa en las regiones es preparar para el empleo, los egresados del presente estudio de caso cuentan ya con un espacio laboral. Segundo, que la universidad está cumpliendo eficientemente con el encargo de preparar profesionales que apliquen los conocimientos adquiridos ya que de la suma de las dos categorías, los de "influencia individual" y los de "influencia profesional" en su mayoría son profesionales trabajando en su entorno. Y tercero, que la voluntad individual prevalece sobre la influencia universitaria en lo general.

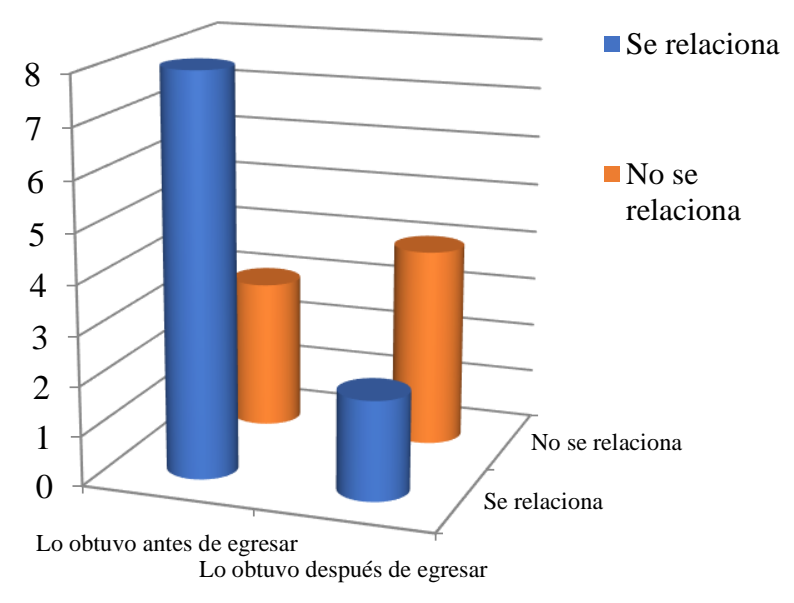

Gráfico 3 Coincidencia del puesto laboral con la formación universitaria

Fuente: información primaria obtenida directamente de las entrevistas

\section{Insuficiencias en la formación}

Las causas que se pudo llegar a conocer a través de la investigación de la que aquí se presentan resultados, se agrupan en tres rubros: las que tienen su origen en aspectos materiales, las que exigen profesionalización del personal, y en las que se solicita una atención especial.
En estos casos, cuando la fuente de información es la opinión de los egresados, deben tomarse con suma reserva por la subjetividad de los individuos; dicen que cada quien comenta cómo le fue en la fiesta en relación de la experiencia particular que tuvo. Algunos alumnos podrán opinar que sus maestros eran de muy mala calidad y es probable que el alumno tenga escaso nivel de comprensión, entonces, del siguiente listado, se deben asumir las afirmaciones con discreción.

\begin{tabular}{|c|c|}
\hline Cualidad & Razones \\
\hline Material & $\begin{array}{l}\text { Falta de herramientas en los } \\
\text { laboratorios. } \\
\text { Los laboratorios de química y en } \\
\text { alimentos no estaban acondicionados. } \\
\text { Algunos laboratorios contaban con } \\
\text { equipo nuevo que nunca se había usado. } \\
\text { No había suficiente material para hacer } \\
\text { las prácticas. } \\
\text { Falta de insumos para los laboratorios. } \\
\text { Hicieron mucha falta mayor cantidad de } \\
\text { prácticas. } \\
\text { Mejor equipamiento en los laboratorios. } \\
\text { Solamente había un laboratorio que era } \\
\text { multifuncional, debería haber más. } \\
\text { Hubo ocasiones en que tuvimos que } \\
\text { improvisar pruebas de laboratorio } \\
\text { utilizando cazuelas. }\end{array}$ \\
\hline Personal & $\begin{array}{l}\text { Hubo marcado ausentismo de } \\
\text { profesores. } \\
\text { Que los profesores hagan más prácticas. } \\
\text { Tuvimos asignaturas donde no } \\
\text { contamos con profesores. } \\
\text { Falta capacitación a profesores. } \\
\text { Que haya una capacitación más } \\
\text { efectiva. } \\
\text { Algunas asignaturas de los primeros } \\
\text { semestres deberían desaparecer porque } \\
\text { no tienen utilidad práctica. }\end{array}$ \\
\hline Atención especial & $\begin{array}{l}\text { No se atiende como debe ser la } \\
\text { impartición de idiomas y tiene una alta } \\
\text { utilidad. } \\
\text { Se debe difundir en la región la } \\
\text { existencia de nuestra carrera para que } \\
\text { los empleadores conozcan de nuestras } \\
\text { posibilidades laborales. } \\
\text { Es necesaria una formación integral, se } \\
\text { debería hacer un esfuerzo en } \\
\text { prepararnos para que nosotros podamos } \\
\text { crear nuestro propio negocio. } \\
\text { Las materias de cálculo diferencial y } \\
\text { álgebra están de más porque no se le ha } \\
\text { encontrado un uso en la vida } \\
\text { profesional. } \\
\text { Se debería de eliminar la asignatura de } \\
\text { tecnología azucarera porque en nuestra } \\
\text { región no existe esa actividad } \\
\text { económica. } \\
\text { Se requiere una mayor formación con la } \\
\text { utilización del idioma inglés. }\end{array}$ \\
\hline
\end{tabular}

Tabla 1 Opinión de los egresados en el origen de las insuficiencias

Fuente: información primaria obtenida directamente de las entrevistas 


\section{Los egresados exitosos en el empleo}

Yolanda $^{6}$ ha contado con cuatro empleos, el primero lo adquirió desde antes de ingresar a la universidad y las actividades que desempeña actualmente coinciden totalmente con su formación académica. Es común pensar que los estudiantes eligen una profesión para que una vez egresado utilice el título para incorporarse al mercado de trabajo en condiciones inmejorables, el caso de Yolanda es a la inversa, al estar conforme con las actividades laborales que desempeña, eligió una carrera universitaria que estuviera acorde a lo que ya hacía. Este es uno de los casos que considero como exitosos porque su experiencia laboral es muy importante puesto que el trabajo en el campo tiene tintes machistas en la región y ella ha luchado contra la corriente en todos los sentidos: porque la mayoría de los egresados son hombres, porque el trabajo en el campo "es para hombres", porque ella se financió su formación universitaria y porque ha transitado por cuatro diferentes experiencias laborales. En la actualidad ofrece asesoría a una empresa mediana en cuestiones de producción en el campo y afirma que nunca ha tenido limitaciones de ningún género.

Genaro tuvo su primera experiencia laboral a la edad de 18 años, poco antes de inscribirse como estudiante en Ingeniería Agroindustrial, ha sido recomendado para trabajar en los tres lugares donde ha entrado, afirma que el certificado universitario fue condición indispensable para obtener el espacio laboral en la fábrica donde él es el responsable de realizar los análisis de laboratorio que se requieren. La empresa es grande y la actividad de responsable de laboratorio que desempeña, la combina con la docencia en una institución del nivel superior. Él mismo costeó sus estudios y considera que su formación fue buena en cuanto que le sirvió para obtener los empleos y que desde su experiencia personal, en la carrera se deberían invertir más recursos para elevar la calidad de los laboratorios de química y de alimentos. Cruz empezó su vida laboral en una empresa familiar, después se ha abierto las puertas por sí mismo, aun cuando considera que hay muchos egresados con los cuales tiene que competir, ha sido exitoso y se desempeña como técnico en un laboratorio de microbiología en alimentos en una empresa grande.
Él mismo se costeó sus estudios y sus padres estudiaron solamente hasta la secundaria. Considera su formación como satisfactoria.

Sonia, la segunda de las mujeres egresadas y con éxito laboral, obtuvo empleo cuando ya estaba estudiando en la universidad, no ha encontrado ningún obstáculo profesional en ninguno de los dos trabajos que ha tenido y en la actualidad es la responsable de un rastro de cerdos mismo que coordina en su totalidad y su obligación principal es la revisión de todo lo relacionado con laboratorios. Considera buena su formación escolar y para el sostenimiento de sus estudios contó con el apoyo de sus padres aun cuando ella ya recibía sueldo.

Salvador es el único egresado hijo de un profesionista. Siempre le llamó la atención lo relacionado con el consumo humano y aunque había hecho solicitud a una institución de educación superior en Aguascalientes, se decidió por la carrera de Ingeniería Agroindustrial en el Centro Universitario de Los Altos. Obtuvo su primer empleo a la edad de 15 años, es decir, antes de ingresar a la universidad, ha trabajado en 5 lugares diferentes, el primero por medio de una recomendación familiar y en el actual ascendió a la jefatura por méritos propios. Es jefe de control de calidad, revisa la materia prima, es el responsable de las calderas, se hace cargo de todo lo relacionado de los análisis microbiológicos y los productos terminados. La empresa es de las más grandes en la región de Los Altos de Jalisco. Afirma que su formación fue muy buena y de lo único que tiene queja en su paso por la universidad es que había equipos nuevos en los laboratorios que no se habían utilizado "como si fueran piezas de museo".

Saúl quiso estudiar la carrera de Química o la de Químico Farmacobiólogo porque desde siempre le había interesado la alimentación, las descartó y se inclinó por la de Ingeniería Agroindustrial porque aquí se analiza y produce, mientras que en aquellas solamente se analiza. Ha trabajado en dos lugares después de haber egresado de la carrera, el primero lo obtuvo en una empresa grande por medio de la recomendación de un amigo; era el responsable del laboratorio de genética en el cual incluso participó como coautor en la publicación de obras, y el segundo es propio y consiste en ofrecer asesorías a empresas.

\footnotetext{
${ }^{6}$ En todos los casos se utilizarán seudónimos para conservar el anonimato. 
Su formación universitaria es aceptable aunque considera que se podría hacer algo para que fuera más integral.

Gonzalo es pionero en estudios universitarios de su familia, tiene 5 hermanos y ninguno cuenta con nivel superior. Sostuvo sus estudios con un trabajo de medio turno que consiguió con el apoyo de un primo y le favoreció mucho porque en esa época murió su padre. Ha trabajado en 5 espacios diferentes, nunca ha tenido problemas por no poderse acoplar al trabajo y utilizar sus saberes universitarios, por el contrario, el poseer el título le ha abierto puertas y actualmente es el encargado de la pasteurización de lácteos y la revisión de la mayor parte de los equipos con los que cuenta la empresa que es de tamaño medio entre las que hay en la región. Califica su formación como buena.

Luis al igual que Gonzalo es pionero en su incorporación a la universidad, tiene 7 hermanos y ninguno ha llegado al nivel superior. Para sostenerse en la carrera fue necesario que un hermano lo ayudara, en este grupo de egresados es el único caso en que se depende de un tutor y no de uno de los padres de familia. Su padre es campesino sin tierras, les trabaja a otras personas y los ingresos no son los suficientes para mantener a universitarios estudiando. $\mathrm{Su}$ primer empleo de una cantidad de puestos que ha tenido y que él calcula entre unos 15 , lo obtuvo cuando vio un anuncio en un periódico local; en la mayor parte de sus incursiones laborales ha ascendido y considera que su principal problema ha sido la falta de experiencia, la universidad debe capacitar más en el trabajo. Su título le ha servido en los últimos puestos y actualmente auxilia a un ingeniero en los procesos productivos que desarrolla la empresa que es grande de tamaño.

Daniel es el último de los casos que considero como de los más exitosos en el ámbito laboral, la escolaridad de sus padres es la enseñanza primaria, sus estudios los sostuvo económicamente por sí mismo, de cuatro hermanos con que cuenta, ninguno se ha inscrito en la universidad. Su primer trabajo lo consiguió cuando tenía solamente 12 años de edad, quedó huérfano de padre y se vio obligado a apoyar a su madre desde que le fue posible.
Se desempeñaba como ayudante en un tendejón y una ocasión una persona mayor se ofreció a contratarlo en otra empresa, ha contado con 6 empleos y en varios de ellos pudo ascender en los puestos.

Se considera afortunado porque nunca ha tenido problemas cuando cambia de un espacio laboral a otro. En la actualidad labora en el área de cárnicos en una empresa pequeña y si bien sus ingresos no son altos, está desempeñando actividades que conoció en su formación universitaria.

\section{Opinión de los empresarios}

Uno de los empresarios que emplea a 12 profesionistas y su industria tiene alcance nacional dice que prefiere contratarlos por medio de recomendaciones porque no confía en los beneficios de las bolsas de trabajo y en las convocatorias abiertas por periódico, él cree más en las relaciones que ha establecido con sus familiares porque eso le ha dado buenos resultados.

Los cuatro aspectos que considera como los más importantes son la honestidad, la disponibilidad, la responsabilidad y el trabajo en equipo. No tiene predisposición por los egresados de algunas universidades y tampoco discrimina por cuestiones de género.

Inclusive, como un beneficio particular, les ofrece apoyo durante cuatro meses para que los egresados terminen sus trámites de titulación en su carrera. Otro empresario que representa un centro laboral de mayores dimensiones que el anterior y de alcance nacional, pertenece al área de la producción animal, ha contratado a 17 profesionistas.

El aspecto que más le interesa es que conozcan de las técnicas que ahí se utilizan; les exige que tengan habilidad en el uso del equipo y mucho conocimiento de su carrera.

Reconoce cinco aptitudes en específico para los egresados del Centro Universitario de Los Altos: accesibilidad, que comparten conocimientos, disponibilidad, se adaptan a los cambios y siempre demuestran ganas de trabajar. 
El responsable del área de los recursos humanos de una tercera empresa describe la misión de su empresa que se considera "líder, con productos competitivos en calidad y precios, buscando el desarrollo integral, a través de una filosofía de servicio" y su visión "Ser la empresa más importante del sector avícola, porcícola y ganadero en el mercado nacional, dedicándonos a producir proteína animal de la mejor calidad para el consumo humano, aplicando innovadores sistemas y tecnología de vanguardia" la compañía es de alcance internacional, se fundó hace más de sesenta años y cuenta con más de 100 trabajadores, contrata a los profesionistas por medio de bolsa de trabajo. Dadas las características de la firma que es de largo alcance, cuenta con áreas donde los profesionistas utilizan sus conocimientos en mercadotecnia, veterinaria, negocios internacionales, comunicación, administración y computación, pero las más importantes están referidas a las ingenierías vinculadas a la producción agropecuaria. Para la elección de los profesionales, les aplican exámenes de conocimiento de su área, deben exponer cuáles son las habilidades con las que cuentan, qué estrategias utilizan en el trabajo, cuáles son los valores con los que cuentan y para ello los entrevistan y los someten a pruebas durante varios días.

No tienen predilección por los egresados de instituciones privadas y los parámetros que aplican en la elección están relacionados con los saberes del área en que se requieren. En particular, con respecto a los egresados del Centro Universitario de Los Altos, aseveran que son personas con ímpetu para salir adelante, cuentan con una mayor capacidad para resolver problemas y son en general innovadores; en contrapartida, consideran que en algunas ocasiones no cuentan con experiencia laboral previa, es el primer lugar al que han acudido para solicitar trabajo.

Otro empresario, dueño de una industria grande con más de mil empleados y con 12 años de antigüedad, afirma que el aspecto más relevante a tomar en cuenta cuando contrata a un profesionista además de que conozca efectivamente de su área, es que no hable mal de otras personas porque eso le da una idea de la honestidad y capacidad que puede desarrollar. Existe un departamento de contrataciones pero él siempre tiene acceso a las entrevistas y siendo el único dueño su decisión es inapelable.
Cuando van en busca de trabajo y hay vacantes en su área, les hacen entrevistas, exámenes psicológicos y algunos filtros entre los que destaca uno que permita estar seguros que el aspirante "se ponga la camiseta" de la empresa y que si maneja trabajadores que no lo vean como un jefe sino que lo hagan sentir como uno más del grupo. Tienen la idea de que la preparación de los egresados de la Universidad de Guadalajara es buena porque a sus alumnos se les prepara para que tengan iniciativa. La última de las empresas de las cuales contamos con el testimonio ya sea de los propietarios o de los responsables de las contrataciones, aunque es un corporativo de 10 firmas, no tiene alcance internacional porque todo lo que transforma se queda en el país aunque afirman que es cuestión de tiempo cuando se dé el siguiente paso.

La guía para la contratación de profesionistas por instrucciones de los propietarios, debe estar acorde con la filosofía de la empresa que es en conjunto: la mejora constante de los procesos, el cumplimiento con la normatividad y legislación aplicable, la adopción de medidas necesarias para hacer uso racional de los recursos naturales, la honestidad, la responsabilidad, el respeto y la colaboración.

Un mecanismo que han utilizado para la contratación de profesionistas es que han recibido una buena cantidad de ellos para realizar sus prácticas profesionales y en ese lapso han tenido la oportunidad de observarlos y contratan a los que han visto que les ofrecen mejores resultados. Un aspecto que consideran en muy alto valor, es que demuestren habilidad para aprender, más que ya cuenten con muchos conocimientos, pero debido a que las áreas de trabajo son muy particulares, no les interesa que ya conozcan todo y lo hayan aprendido en la universidad, sino verlos que tienen mucho entusiasmo por aprender las cosas nuevas en la empresa.

Han observado que varios de los prospectos se resisten a salir de "su área de confort" y eso sí es dañino; "es imposible que cuando terminan la universidad puedan conocer todo lo que se necesita en el trabajo" pero los solicitantes que muestran mayor disponibilidad para aprender y para desprenderse de su área de comodidad son los que se quedan. 


\section{Conclusiones}

La inserción laboral de los profesionales de la ingeniería agroindustrial es exitosa por varios motivos, primero, porque la totalidad de los consultados contaba con un empleo al momento de la entrevista; segundo, porque proviniendo todos de un nivel económico bajo, ascendieron socialmente y ahora son universitarios insertados en las actividades propias de trabajadores calificados. Tercero, porque la inmensa mayoría de ellos se solventaron económicamente sus estudios y al interior de sus familias el índice de familiares inscritos en la universidad es muy bajo. Cuarto, porque a pesar de que el medio laboral donde se desenvuelven es "para hombres" varias de las egresadas se han desenvuelto exitosamente. Quinto, porque han sido bien recibidos por los contratantes $\mathrm{y}$ reconocen en ellos su calidad, constancia y compromiso con las empresas.

La Teoría del Capital Cultural es apropiada para explicar el ascenso de los ingenieros agroindustriales entrevistados. Se pudieron demostrar sus principales postulados por las siguientes características: los egresados provienen de familias de bajos recursos y pudieron ascender en la escala social, por lo tanto se comprueba que la decisión de inscribirse en la universidad para "invertirse" en ellos mismos y convertirse al egreso en trabajadores calificados fue la apropiada. No existe alguna otra influencia externa que haya afectado en su trayectoria escolar en forma positiva y que ponga en duda los preceptos de la Teoría del Capital Humano como el atributo principal. La gran mayoría cuenta con un empleo que coincide plenamente con su formación universitaria y la mayor parte de las personas que conformaron el objeto de estudio, se incorporaron a los estudios profesionales cuando ya estaban trabajando; lo anterior significa para los alcances de esta investigación, que el haber optado por una mayor capacitación en el trabajo al interior de las aulas universitarias significó el motivo más trascendente en su desarrollo personal. Para la Teoría del Capital Humano, este punto de inflexión es el más determinante. Los ingresos económicos que obtienen como jefes, coordinadores o responsables de área, son mayores que los devengados por los trabajadores no calificados en los espacios laborales donde se ubican.
En referencia a las carencias en la formación universitaria, los funcionarios deben poner puntual atención en la infraestructura porque es donde se expresaron las principales preocupaciones, en especial sobre la carencia de materiales y equipos en los laboratorios. Los ingenieros agroindustriales cuentan con una formación muy marcada en la transformación de alimentos y el no contar con los medios idóneos para el aprendizaje, es obvio que no contarán con las destrezas cuando se enfrenten con la realidad laboral. Otro rubro es el de la formalidad y constancia en la docencia, los mecanismos de supervisión deben ser eficaces; las actualizaciones en el Plan de Estudios deben estar al día.

\section{Referencias}

(2010). XI Censos de población y vivienda. México: Instituto Nacional de Estadística y Geografía.

Aronson, P. P. (2007). El retorno de la teoría del capital humano. Red de Revistas Científicas de América Latina y el Caribe, España y Portugal, 9-26.

Bowles, S., y Gintis, H. (1975). El rpoblema de la teoría del capital humano: una crítica marxista. american Economic Review, vol LXV, núm. 2, 220-228.

Briceño Mosquera, A. (2011). La educación y su efecto en la formación de capital humano y en el desarrollo económico de los países. Apuntes del CENES. Volumen 30, número 51., 45-59.

Garrido Trejo, C. (2007). La educación desde la teoría del capital humano y el otro. Red de Revistas Científicas de América Latina y el Caribe, España y Portugal., 73-80.

González Pérez, C., y Machaen López, L. E. (2015). Seguimiento a egresados de la Carrera de Negocios Internacionales del Centro Universitario de Los Altos de la Universidad de Guadalajara. Revista Iberoamericana de Contaduría, Economía y Administración.

González Pérez, C., y Ortega Cervantes, G. (2015). Empleabilidad de los egresados de la Carrera de Contaduría Pública. Revista Iberoamericana para la Investigación y el Desarrollo Educativo, 1-21. 
Kumar Sen, A. (1998). Capital humano y capacidad humana. Cuadernos de Economía, 67-72.

Leyva López, S., y Cárdenas Almagro, A. (2002). Economía de la educación: capital humano y rendimiento educativo. Análisis Económico, 79-106.

Villalobos Monroy, G., y Pedroza Flores, R. (julio- diciembre, 2009). Perspectiva de la Teoría del Capital Humano acerca de la relación entre educación y desarrollo económico. Tiempo de educar, columen 10, número 20, 273-306. 